\title{
Bi-matrix game in bifuzzy environment
}

\author{
Sankar Kumar Roy ${ }^{1 *}$ and Prasanta Mula²
}

\author{
*Correspondence: \\ sankroy2006@gmail.com \\ ${ }^{1}$ Department of Applied \\ Mathematics with Oceanology and \\ Computer Programming, \\ Vidyasagar University, Midnapore, \\ West Bengal 721102, India \\ Full list of author information is \\ available at the end of the article
}

\begin{abstract}
In this paper, a new concept of bifuzzy bi-matrix game is introduced where all elements of the payoff matrices are characterized by bifuzzy variables. The uncertainties of entries of payoff matrices (bifuzzy variables) are measured by bifuzzy measure known as Chance measure. Combining the bifuzzy set theory and bi-matrix game theory, the solution concept of bifuzzy bi-matrix game theory is introduced. The quadratic programming problem plays the major role to solve bifuzzy bi-matrix game. In order to show the applicability and feasibility of our proposed method, a real-life bi-matrix game problem is considered and solved.
\end{abstract}

Keywords: Bifuzzy variable; Expected value operator; Chance measure; Quadratic programming; Nash equilibrium strategy

\section{Introduction}

Game theory [1] is a bag of analytical tools designed to help us understand the phenomena that we observed when decision-makers interact. The models of game theory are highly abstract representations of classes of real-life situations. The basic assumption is that the decision-makers pursued well-defined exogenous objectives and take into account their knowledge and expectations of other decision-maker's behavior.

A game is a formal description of a strategic situation. Game theory is applied whenever the actions of several agents are interdependent. These agents may be individuals, groups, firms, or any combination of these. The concept of game theory provides a language to formulate, structure, analyze, and understand the strategic scenario. A payoff is a number, also called utility, that reflects the desirability of an outcome to a player, for whatever reason. When the outcomes are uncertain variables, payoffs are usually weighted with their uncertainty. The expected payoff incorporates the player's attitude toward risk. A game in strategic form, also called normal form, is a compact representation of a game in which players simultaneously choose their strategies. The resulting payoffs are presented in a table with a cell for each strategy combination. In a game in strategic form, a strategy is one of the given possible actions of a player. In an extensive game, a strategy is a complete plan of choices, one for each decision point of the player.

In game theory, the players have to choose appropriate strategy to optimize their gain. There have been different methodologies in bi-matrix game theory to optimize the solution, a well-known technique is Wolfe's modified simplex method. In traditional game theory, the elements of payoff matrix are considered as real numbers, and this basically depends upon the gain of the players. In recent years, attempts have been made to extend

(C) 2013 Roy and Mula; licensee Springer. This is an Open Access article distributed under the terms of the Creative Commons

Attribution License (http://creativecommons.org/licenses/by/2.0), which permits unrestricted use, distribution, and reproduction in any medium, provided the original work is properly cited. 
the results of crisp game theory to the fuzzy games [2-7]. In credibilistic bi-matrix game [8], the payoff elements are fuzzy variables. To find the optimum solution, applying the fuzzy measurable function known as credibility defined by Liu [9] in 2002. Using credibility theory and game theory, the credibilistic bi-matrix game is converted into crisp quadratic programming problem which depends upon the confidence level of the players' payoff matrix. In bifuzzy matrix game [10], the elements of the payoff matrix are bifuzzy variables, and its uncertainty is measured by bifuzzy measurable function known as Chance measure introduced by Liu [9]. Zhou and Liu [11] introduced the concept of chance distribution for bifuzzy variable, and they also introduced the expected value operator and its linearity on bifuzzy variable.

In many cases, fuzzy variable is not sufficient to handle some type of real-life practical problems on bi-matrix game. As a result, a new type of variable is incorporated which known as bifuzzy variable of bifuzzy set theory. Due to this reason, the elements of payoff matrices (bi-matrix game) are considered as bifuzzy variables in this paper. In fuzzy variables, the bounds are real numbers whereas in bifuzzy variable, the bounds are fuzzy variables and the uncertainty of bifuzzy variable is measured by chance measure. Combining the bifuzzy set theory and bi-matrix game, bifuzzy bi-matrix game has been formulated. The optimum solution of bifuzzy bi-matrix game, depending upon their confidence levels, is discussed in this paper. Finally, a practical example is included to explain the proposed methodology, and then, solution procedure has been discussed.

\section{Preliminaries}

Given a universe $\Theta, P(\Theta)$ is the power set of $\Theta$, and a set function Pos defines on $P(\Theta)$ which is called a possibility measure if it satisfies the following conditions

1. $\operatorname{Pos}(\phi)=0, \phi$ is an empty set.

2. $\operatorname{Pos}(\Theta)=1$.

3. $\operatorname{Pos}\left(\cup_{i \in I} A_{i}\right)=\sup _{i \in I} \operatorname{Pos}\left(A_{i}\right)$ for any subclass $\left\{A_{i} \mid i \in I\right\}$ of $P(\Theta)$.

The necessity measure of a set $A$ is defined as the impossibility of the opposite set $A^{c}$. Then, the necessity measure of $A$ is defined by

$$
\operatorname{Nec}(A)=1-\operatorname{Pos}\left(A^{c}\right) .
$$

The triplet $(\Theta, P(\Theta)$, Pos) is usually called a possibility space, which is also called a pattern space. In addition, a self dual set function is called credibility measure $[9,12]$ and defined as follows:

$$
\operatorname{Cr}(A)=\frac{1}{2}\left[\operatorname{Pos}(A)+1-\operatorname{Pos}\left(A^{c}\right)\right]
$$

for any $A \in P(\Theta)$ where $A^{c}$ is the complement of $A$.

A fuzzy variable $\xi$ is defined as a function from a credibility space $(\Theta, P(\Theta), \mathrm{Cr})$ to the set of real numbers. Based on credibility measure, the expected value of fuzzy variable $\xi$ is defined as:

$$
E[\xi]=\int_{0}^{\infty} \operatorname{Cr}\{\xi \geq r\} d r-\int_{-\infty}^{0} \operatorname{Cr}\{\xi \leq r\} d r
$$

provided that at least one of the two integrals is finite. 
Example 1. (Liu and Lui [13]) Let $\xi=(a, b, c, d)$ be a trapezoidal fuzzy variable with $c \leq a<b \leq d$. Then, we have

$$
E[\xi]=\frac{1}{4}(a+b+c+d)
$$

Given a credibility space $(\Theta, P(\Theta), \mathrm{Cr})$ which is complete, we obtain the definition of bifuzzy variable as follows

Definition 1. (Liu [9]) A bifuzzy variable is a function from the credibility space $(\Theta, P(\Theta), C r)$ to the set of fuzzy variables.

Definition 2. (Liu [9]) Let $(\Theta, P(\Theta)$, Cr $)$ be a credibility space. A map $\zeta=\left(\zeta_{1}, \zeta_{2}, \ldots, \zeta_{n}\right)^{T}$ : $\Theta \rightarrow F_{\alpha}{ }^{n}$ is said to be an n-array bifuzzy vector iffor any Borel subset $\mathbf{B}$ of $\bar{\Re}^{n}$, the function

$$
\operatorname{Cr}\left\{\theta \in \Theta \mid \zeta_{\theta}\left(\theta^{\prime}\right) \in \mathbf{B}\right\}
$$

is treated as measurable with respect to $\theta$, as $n=1$, where $\zeta$ is called a bifuzzy variable.

Definition 3. (Liu [9]) An n-dimensional bifuzzy vector is a function from the credibility space $(\Theta, P(\Theta), C r)$ to the set of $n$-dimensional fuzzy vector.

Theorem 1. (Liu [9]) Assume that $\zeta$ is a bifuzzy variable. Then for any set $\mathbf{B}$ of $\Re$, we have

(a) The possibility $\operatorname{Pos}\{\zeta(\theta) \in \mathbf{B}\}$ is a fuzzy variable.

(b) The necessity $\operatorname{Nec}\{\zeta(\theta) \in \mathbf{B}\}$ is a fuzzy variable.

(c) The credibility $\operatorname{Cr}\{\zeta(\theta) \in \mathbf{B}\}$ is a fuzzy variable.

Definition 4. (Liu [9]) Suppose $\zeta$ is a bifuzzy variable, the expected value of $\zeta$ is a real number defined as,

$$
E[\zeta]=\int_{0}^{\infty} \operatorname{Cr}\{\theta \in \Theta \mid E[\zeta(\theta)] \geq r\} d r-\int_{-\infty}^{0} \operatorname{Cr}\{\theta \in \Theta \mid E[\zeta(\theta)] \leq r\} d r
$$

provided that at least one of the two integrals is finite.

Theorem 2. (Liu [9]) Let $\zeta$ be a bifuzzy variable. If the expected value $E[\zeta(\theta)]$ is finite for each $\theta$, then $E[\zeta(\theta)]$ is a fuzzy variable.

Definition 5. (Zhou and Liu [11]) Assume that $\zeta$ and $\rho$ are bifuzzy variables with finite expected values. If for each $\theta \in \Theta$, the fuzzy variables $\zeta(\theta)$ and $\rho(\theta)$ are independent, and $E[\zeta(\theta)]$ and $E[\rho(\theta)]$ are independent fuzzy variables, then for any real numbers $a$ and $b$, we have

$$
E[a \zeta+b \rho]=a E[\zeta]+b E[\rho]
$$

Example 2. Let $\zeta=\left(\xi_{1}, \xi_{2}, \xi_{3}, \xi_{4}\right)$ be a bifuzzy variable with independent trapezoidal fuzzy variable $\xi_{i}=\left(a_{i}, b_{i}, c_{i}, d_{i}\right)$ for $i=1,2,3,4$. Then we have

$$
\begin{aligned}
E[\zeta]= & \frac{1}{4} E\left[\xi_{1}+\xi_{2}+\xi_{3}+\xi_{4}\right]=\frac{1}{4}\left[E\left[\xi_{1}\right]+E\left[\xi_{2}\right]+E\left[\xi_{3}\right]+E\left[\xi_{4}\right]\right] \\
= & \frac{1}{4}\left[\frac{1}{4}\left(a_{1}+b_{1}+c_{1}+d_{1}\right)+\frac{1}{4}\left(a_{2}+b_{2}+c_{2}+d_{2}\right)+\frac{1}{4}\left(a_{3}+b_{3}+c_{3}+d_{3}\right)\right. \\
& \left.+\frac{1}{4}\left(a_{4}+b_{4}+c_{4}+d_{4}\right)\right] \\
= & \frac{1}{16}\left(a_{1}+b_{1}+c_{1}+d_{1}+a_{2}+b_{2}+c_{2}+d_{2}+a_{3}+b_{3}+c_{3}+d_{3}+a_{4}+b_{4}+c_{4}+d_{4}\right)
\end{aligned}
$$

where the fuzzy variable $\xi_{i}=\left(a_{i}, b_{i}, c_{i}, d_{i}\right)$ such that $c_{i} \leq a_{i}<b_{i} \leq d_{i}$ for $i=1,2,3,4$. 
Definition 6. (Liu [9]) Let $\zeta$ be a bifuzzy variable and $\mathbf{B}$ be a set of real number $\Re$. Then, the chance measure denoted by Ch of bifuzzy event $\zeta \in \mathbf{B}$ is a function from $(0,1]$ to $[0,1]$ defined as,

$$
\operatorname{Ch}\{\zeta \in B\}(\alpha)=\sup _{C} r\{A\} \geq \operatorname{\alpha inf}_{\theta \in A} \operatorname{Cr}\{\zeta(\theta) \in \mathbf{B}\}
$$

Theorem 3. (Zhou and Liu [11]) Let $\zeta$ be a bifuzzy variable and $\mathbf{B}$ be a Borel set of $\Re$. For any given $\alpha^{*}>0.5$, we write $\delta^{*}=\operatorname{Ch}\{\zeta \in \mathbf{B}\}\left(\alpha^{*}\right)$. Then, we have

$$
\operatorname{Cr}\left\{\theta \in \Theta \mid \operatorname{Cr}\{\zeta(\theta) \in \mathbf{B}\} \geq \delta^{*}\right\} \geq \alpha^{*}
$$

Definition 7. (Liu [9]) Let $\zeta$ be a bifuzzy variable and $\alpha, \delta \in(0,1]$. Then

$$
\zeta_{\text {sup }}(\alpha, \delta)=\sup \{w \mid C h\{\zeta \geq w\}(\alpha) \geq \delta\}
$$

is called the $(\alpha, \delta)$-optimistic value to $\zeta$ and

$$
\zeta_{\text {inf }}(\alpha, \delta)=\inf \{w \mid C h\{\zeta \leq w\}(\alpha) \geq \delta\}
$$

is called the $(\alpha, \delta)$-pessimistic value to $\zeta$

Theorem 4. (Zhou and Liu [11]) Let $\zeta$ be a bifuzzy variable. Assume that $\zeta_{\text {sup }}(\alpha, \delta)$ is the $(\alpha, \delta)$-optimistic value of $\zeta$ and $\zeta_{\text {inf }}(\alpha, \delta)$ is the $(\alpha, \delta)$-pessimistic value to $\zeta$. If $\alpha>0.5$ and $\delta>0.5$ then we have,

$$
C h\left\{\zeta \leq \zeta_{\text {inf }}(\alpha, \delta)\right\}(\alpha) \geq \delta, \quad C h\left\{\zeta \geq \zeta_{\text {sup }}(\alpha, \delta)\right\}(\alpha) \geq \delta
$$

Example 3. Let us consider the trapezoidal fuzzy variable $\xi=(a, b, c, d)$ with the crisp relation $a \leq b<c \leq d$. Then, the fuzzy measures namely possibility, necessity, and credibility are defined by Liu [9] as follows:

$$
\begin{gathered}
\operatorname{Pos}\{\xi \leq 0\}= \begin{cases}1 & \text { if } b \leq 0, \\
\frac{a}{a-b} & \text { if } a \leq 0 \leq b, \\
0 & \text { if otherwise. }\end{cases} \\
\operatorname{Nec}\{\xi \leq 0\}= \begin{cases}1 & \text { if } d \leq 0, \\
\frac{c}{c-d} & \text { if } c \leq 0 \leq d, \\
0 & \text { if otherwise. }\end{cases} \\
\operatorname{Cr}\{\xi \leq 0\}= \begin{cases}1 & \text { if } d \leq 0, \\
\frac{2 c-d}{2(c-d)} & \text { if } c \leq 0 \leq d, \\
\frac{1}{2} & \text { if } b \leq 0 \leq c, \\
\frac{a}{2(a-b)} & \text { if } a \leq 0 \leq b, \\
0 & \text { if otherwise. }\end{cases}
\end{gathered}
$$

Theorem 5. (Liu [9]) Let us consider the trapezoidal fuzzy variable $\xi=(a, b, c, d)$ and confidence level $\alpha \in(0,1]$, we have

(1) $\operatorname{Pos}\{\xi \leq 0\} \geq \alpha$, if and only if $(1-\alpha) a+\alpha b \leq 0$

(2) $\operatorname{Nec}\{\xi \leq 0\} \geq \alpha$, if and only if $(1-\alpha) c+\alpha d \leq 0$

(3) when $\alpha \leq \frac{1}{2}, \operatorname{Cr}\{\xi \leq 0\} \geq \alpha$, if and only if $(1-2 \alpha) a+2 \alpha b \leq 0$

(4) when $\alpha>\frac{1}{2}, \operatorname{Cr}\{\xi \leq 0\} \geq \alpha$, if and only if $(2-2 \alpha) c+(2 \alpha-1) d \leq 0$ 
Theorem 6. (Liu [9]) Let $\xi_{k}=\left(a_{k}, b_{k}, c_{k}, d_{k}\right)$ be trapezoidal fuzzy variables for $k=$ $1,2, \cdots, n$ and a function $g(x, \xi)$ can be written as,

$$
g(x, \xi)=h_{1}(x) \xi_{1}+h_{2}(x) \xi_{2}+\cdots+h_{n}(x) \xi_{n}+h_{0}(x)
$$

If two functions are defined as $h_{k}^{+}(x)=h_{k}(x) \vee 0$ and $h_{k}^{-}(x)=-h_{k}(x) \wedge 0$ for $k=$ $1,2, \cdots, n$, Then, there exists a confidence level $\alpha \in(0,1]$,

(1) when $\alpha<1 / 2, \operatorname{Cr}\{g(x, \xi) \leq 0\} \geq \alpha$, if and only if,

$$
(1-2 \alpha) \sum_{k=1}^{n}\left[a_{k} h_{k}^{+}(x)-d_{k} h_{k}^{-}(x)\right]+2 \alpha \sum_{k=1}^{n}\left[b_{k} h_{k}^{+}(x)-c_{k} h_{k}^{-}(x)\right]+h_{0}(x) \leq 0
$$

(2) when $\alpha \geq 1 / 2, \operatorname{Cr}\{g(x, \xi) \leq 0\} \geq \alpha$, if and only if,

$$
(2-2 \alpha) \sum_{k=1}^{n}\left[c_{k} h_{k}^{+}(x)-b_{k} h_{k}^{-}(x)\right]+(2 \alpha-1) \sum_{k=1}^{n}\left[d_{k} h_{k}^{+}(x)-a_{k} h_{k}^{-}(x)\right]+h_{0}(x) \leq 0
$$

Theorem 7. Let $\zeta_{k}=\left(\xi_{1 k}, \xi_{2 k}, \xi_{3 k}, \xi_{4 k}\right)$ be bifuzzy variables for $k=1,2, \cdots$, n with trapezoidal fuzzy variable $\xi_{m k}=\left(a_{m k}, b_{m k}, c_{m k}, d_{m k}\right)$ for $m=1,2,3,4$ and a function $g(x, \zeta)$ can be written as,

$$
g(x, \zeta)=h_{1}(x) \zeta_{1}+h_{2}(x) \zeta_{2}+\cdots+h_{n}(x) \zeta_{n}+h_{0}(x)
$$

If two functions are defined as $h_{k}^{+}(x)=h_{k}(x) \vee 0$ and $h_{k}^{-}(x)=-h_{k}(x) \wedge 0$ for $k=$ $1,2, \cdots, n$. Then, there exists a confidence level $(\alpha, \delta) \in(0,1]$,

(1) when $\delta<1 / 2, \operatorname{Ch}\{g(x, \zeta) \leq 0\}(\alpha) \geq \delta$, if and only if,

$$
(1-2 \delta) P_{11}+2 \delta P_{12}+h_{0}(x) \leq 0
$$

(2) when $\delta \geq 1 / 2, \operatorname{Ch}\{g(x, \zeta) \leq 0\}(\alpha) \geq \delta$, if and only if,

$$
(2-2 \delta) P_{21}+(2 \delta-1) P_{22}+h_{0}(x) \leq 0
$$

where $P_{11}$ defined as,

(1) when $\alpha<1 / 2$, if and only if,

$$
P_{11}=(1-2 \alpha) \sum_{k=1}^{n}\left[a_{1 k} h_{k}^{+}(x)-d_{4 k} h_{k}^{-}(x)\right]+2 \alpha \sum_{k=1}^{n}\left[b_{1 k} h_{k}^{+}(x)-c_{4 k} h_{k}^{-}(x)\right]
$$

(2) when $\alpha \geq 1 / 2$, if and only if,

$$
P_{11}=(2-2 \alpha) \sum_{k=1}^{n}\left[c_{1 k} h_{k}^{+}(x)-b_{4 k} h_{k}^{-}(x)\right]+(2 \alpha-1) \sum_{k=1}^{n}\left[d_{1 k} h_{k}^{+}(x)-a_{4 k} h_{k}^{-}(x)\right]
$$

where $P_{12}$ defined as,

(1) when $\alpha<1 / 2$, if and only if,

$$
P_{12}=(1-2 \alpha) \sum_{k=1}^{n}\left[a_{2 k} h_{k}^{+}(x)-d_{3 k} h_{k}^{-}(x)\right]+2 \alpha \sum_{k=1}^{n}\left[b_{2 k} h_{k}^{+}(x)-c_{3 k} h_{k}^{-}(x)\right]
$$


(2) when $\alpha \geq 1 / 2$, if and only if,

$$
P_{12}=(2-2 \alpha) \sum_{k=1}^{n}\left[c_{2 k} h_{k}^{+}(x)-b_{3 k} h_{k}^{-}(x)\right]+(2 \alpha-1) \sum_{k=1}^{n}\left[d_{2 k} h_{k}^{+}(x)-a_{3 k} h_{k}^{-}(x)\right]
$$

where $P_{21}$ defined as,

(1) when $\alpha<1 / 2$, if and only if,

$$
P_{21}=(1-2 \alpha) \sum_{k=1}^{n}\left[a_{3 k} h_{k}^{+}(x)-d_{2 k} h_{k}^{-}(x)\right]+2 \alpha \sum_{k=1}^{n}\left[b_{3 k} h_{k}^{+}(x)-c_{2 k} h_{k}^{-}(x)\right]
$$

(2) when $\alpha \geq 1 / 2$, if and only if,

$$
P_{21}=(2-2 \alpha) \sum_{k=1}^{n}\left[c_{3 k} h_{k}^{+}(x)-b_{2 k} h_{k}^{-}(x)\right]+(2 \alpha-1) \sum_{k=1}^{n}\left[d_{3 k} h_{k}^{+}(x)-a_{2 k} h_{k}^{-}(x)\right]
$$

where $P_{22}$ defined as,

(1) when $\alpha<1 / 2$, if and only if,

$$
P_{22}=(1-2 \alpha) \sum_{k=1}^{n}\left[a_{4 k} h_{k}^{+}(x)-d_{1 k} h_{k}^{-}(x)\right]+2 \alpha \sum_{k=1}^{n}\left[b_{4 k} h_{k}^{+}(x)-c_{1 k} h_{k}^{-}(x)\right]
$$

(2) when $\alpha \geq 1 / 2$, if and only if,

$$
P_{22}=(2-2 \alpha) \sum_{k=1}^{n}\left[c_{4 k} h_{k}^{+}(x)-b_{1 k} h_{k}^{-}(x)\right]+(2 \alpha-1) \sum_{k=1}^{n}\left[d_{4 k} h_{k}^{+}(x)-a_{1 k} h_{k}^{-}(x)\right]
$$

Proof. Since $h_{k}^{+}(x)$ and $h_{k}^{-}(x)$ both are nonnegative functions and $h_{k}(x)=h_{k}^{+}(x)-h_{k}^{-}(x)$, thus we have

$$
\begin{aligned}
g(x, \zeta) & =\sum_{k=1}^{n} h_{k}(x) \zeta_{k}+h_{0}(x) \\
& =\sum_{k=1}^{n}\left[h_{k}^{+}(x)-h_{k}^{-}(x)\right] \zeta_{k}+h_{0}(x) \\
& \left.=\sum_{k=1}^{n}\left[h_{k}^{+}(x) \zeta_{k}+h_{k}^{-}(x) \zeta_{k}^{\prime}\right)\right]+h_{0}(x)
\end{aligned}
$$

where $\zeta_{k}^{\prime}=\left(-\xi_{4 k},-\xi_{3 k},-\xi_{2 k},-\xi_{1 k}\right)$ for $k=1,2, \cdots, n$ with $-\xi_{m k}=\left(-d_{m k},-c_{m k}\right.$, $\left.-b_{m k},-a_{m k}\right)$ for $m=1,2,3,4$. In the function $g(x, \zeta)$, the addition and multiplication on bifuzzy variables is determined by quadruple as follows,

$$
g(x, \zeta)=\left(\begin{array}{c}
\sum_{k=1}^{n}\left[\xi_{1 k} h_{k}^{+}(x)-\xi_{4 k} h_{k}^{-}(x)\right]+h_{0}(x) \\
\sum_{k=1}^{n}\left[\xi_{2 k} h_{k}^{+}(x)-\xi_{3 k} h_{k}^{-}(x)\right]+h_{0}(x) \\
\sum_{k=1}^{n}\left[\xi_{3 k} h_{k}^{+}(x)-\xi_{2 k} h_{k}^{-}(x)\right]+h_{0}(x) \\
\sum_{k=1}^{n}\left[\xi_{4 k} h_{k}^{+}(x)-\xi_{1 k} h_{k}^{-}(x)\right]+h_{0}(x)
\end{array}\right)^{T}
$$


i.e.,

$$
g(x, \zeta)=\left(\begin{array}{c}
\sum_{k=1}^{n}\left[a_{1 k} h_{k}^{+}(x)-d_{4 k} h_{k}^{-}(x)\right]+h_{0}(x) \\
\sum_{k=1}^{n}\left[b_{1 k} h_{k}^{+}(x)-c_{4 k} h_{k}^{-}(x)\right]+h_{0}(x) \\
\sum_{k=1}^{n}\left[c_{1 k} h_{k}^{+}(x)-b_{4 k} h_{k}^{-}(x)\right]+h_{0}(x) \\
\sum_{k=1}^{n}\left[d_{1 k} h_{k}^{+}(x)-a_{4 k} h_{k}^{-}(x)\right]+h_{0}(x) \\
\sum_{k=1}^{n}\left[a_{2 k} h_{k}^{+}(x)-d_{3 k} h_{k}^{-}(x)\right]+h_{0}(x) \\
\sum_{k=1}^{n}\left[b_{2 k} h_{k}^{+}(x)-c_{3 k} h_{k}^{-}(x)\right]+h_{0}(x) \\
\sum_{k=1}^{n}\left[c_{2 k} h_{k}^{+}(x)-b_{3 k} h_{k}^{-}(x)\right]+h_{0}(x) \\
\sum_{k=1}^{n}\left[d_{2 k} h_{k}^{+}(x)-a_{3 k} h_{k}^{-}(x)\right]+h_{0}(x) \\
\sum_{k=1}^{n}\left[a_{3 k} h_{k}^{+}(x)-d_{2 k} h_{k}^{-}(x)\right]+h_{0}(x) \\
\sum_{k=1}^{n}\left[b_{3 k} h_{k}^{+}(x)-c_{2 k} h_{k}^{-}(x)\right]+h_{0}(x) \\
\sum_{k=1}^{n}\left[c_{3 k} h_{k}^{+}(x)-b_{2 k} h_{k}^{-}(x)\right]+h_{0}(x) \\
\sum_{k=1}^{n}\left[d_{3 k} h_{k}^{+}(x)-a_{2 k} h_{k}^{-}(x)\right]+h_{0}(x) \\
\sum_{k=1}^{n}\left[a_{4 k} h_{k}^{+}(x)-d_{1 k} h_{k}^{-}(x)\right]+h_{0}(x) \\
\sum_{k=1}^{n}\left[b_{4 k} h_{k}^{+}(x)-c_{1 k} h_{k}^{-}(x)\right]+h_{0}(x) \\
\sum_{k=1}^{n}\left[c_{4 k} h_{k}^{+}(x)-b_{1 k} h_{k}^{-}(x)\right]+h_{0}(x) \\
\sum_{k=1}^{n}\left[d_{4 k} h_{k}^{+}(x)-a_{1 k} h_{k}^{-}(x)\right]+h_{0}(x)
\end{array}\right)^{T}
$$

Depending upon the confidence level of $(\delta, \alpha)$, we can separate the above constraints using Theorem 6.

\section{Bi-matrix game}

In this subsection, let us consider the bi-matrix game whose payoff elements are characterized by real numbers. Let $X \equiv\{1,2, \cdots, m\}$ be a set of strategies for the player I and $Y \equiv\{1,2, \cdots, n\}$ be a set of strategies for player II. Let $R^{n}$ be the $n$-dimensional Euclidean space and $R_{+}^{n}$ be its non-negative orthant. Here, $e^{T}$ be a vector of element ' 1 ' whose dimension is specified as per specific context. Mixed strategies of players I and II are represented by $S^{X}=\left\{x \in R_{+}^{m}, e^{T} x=1\right\}$ and $S^{Y}=\left\{y \in R_{+}^{n}, e^{T} y=1\right\}$, respectively.

By considering real numbers $a_{i j}$ and $b_{i j}$ as the expected reward for the players I and II corresponding to the proposed strategies $i$ and $j$, respectively. Then, the bi-matrix game can be defined as follows:

$$
(A, B)=\left(\begin{array}{cccc}
\left(a_{11}, b_{11}\right) & \left(a_{12}, b_{12}\right) & \ldots & \left(a_{1 n}, b_{1 n}\right) \\
\left(a_{21}, b_{21}\right) & \left(a_{22}, b_{22}\right) & \ldots & \left(a_{2 n}, b_{2 n}\right) \\
\cdot & \cdot & \ldots & \cdot \\
\cdot & \cdot & \ldots & \cdot \\
\cdot & \cdot & \ldots & \cdot \\
\left(a_{m 1}, b_{m 1}\right) & \left(a_{m 2}, b_{m 2}\right) & \ldots & \left(a_{m n}, b_{m n}\right)
\end{array}\right)
$$

Definition 8. (Basar and Olsder [14]) A pair $\left(x^{*}, y^{*}\right) \in S^{X} \times S^{Y}$ is said to be Nash equilibrium strategy of the bi-matrix game $B G=\left(S^{X}, S^{Y}, A, B\right)$ if,

$$
\begin{aligned}
x^{T} A y^{*} \leq x^{* T} A y^{*}, \forall x \in S^{X} \\
\text { and } \quad x^{* T} B y \leq x^{* T} B y^{*}, \forall y \in S^{Y}
\end{aligned}
$$


Theorem 8. (Basar and Olsder [14]) Let BG $=\left(S^{X}, S^{Y}, A, B\right)$ be given bi-matrix game. A necessary and sufficient condition that $\left(x^{*}, y^{*}\right)$ be an equilibrium strategy of $B G$ is that it is a solution of the following quadratic programming problem,

$$
\begin{aligned}
\max x^{T}(A+B) y-v-w & \\
\text { subject to, } \quad A y-v e & \leq 0 \\
B^{T} x-w e & \leq 0 \\
e^{T} x-1 & =0 \\
e^{T} y-1 & =0 \\
v, w & \in \Re \\
x, y & \geq 0
\end{aligned}
$$

Lemma 1. If $v^{*}$ be the value of the game for player I where $v^{*}$ is given by

$$
v^{*}=x^{* T} A y^{*}=\max _{x}\left\{x^{T} A y^{*}: e^{T} x=1, x \geq 0\right\}
$$

and $w^{*}$ is the value of the game for player II where $w^{*}$ is given by

$$
w^{*}=x^{* T} B y^{*}=\max _{y}\left\{x^{* T} B y: e^{T} y=1, y \geq 0\right\}
$$

And if we assume that $x_{i}{ }^{\prime}=\frac{x_{i}}{w}$ and $y_{j}{ }^{\prime}=\frac{y_{j}}{v}$ ( for $\left.i=1,2, \cdots, m ; j=1,2, \cdots, n\right)$. Then, the above Theorem 8 reduces into the following quadratic programming problem,

$$
\begin{gathered}
\max e^{T} x^{\prime}+e^{T} y^{\prime}+x^{\prime T}(A+B) y^{\prime} \\
\text { subject to, } \quad \sum_{j=1}^{n} a_{i j} y_{j}^{\prime} \leq 1 \text { for } i=1,2, \cdots, m \\
\sum_{i=1}^{m} b_{i j} x_{i}^{\prime} \leq 1 \text { for } j=1,2, \cdots, n \\
x^{\prime}, y^{\prime} \geq 0
\end{gathered}
$$

where $\left(x^{*}, y^{*}\right)$ and $\left(v^{*}, w^{*}\right)$ are the Nash equilibrium strategy and outcome of the bi-matrix game $B G$.

Proof. Since $\left(x^{*}, y^{*}\right) \in S^{X} \times S^{Y}$ be the Nash equilibrium strategy of the bi-matrix game $B G$ if and only if $x^{*}$ and $y^{*}$ are simultaneously solutions of the following two problems,

$$
\left\{\begin{array}{cc}
\max & x^{T} A y^{*} \\
\text { subject to, } & e^{T} x=1 \\
& x \geq 0 .
\end{array}\right.
$$

and

$$
\left\{\begin{aligned}
\max & x^{* T} B y \\
\text { subject to, } & e^{T} y=1 \\
& y \geq 0 .
\end{aligned}\right.
$$

Here, $\left(x^{*}, y^{*}\right) \in S^{X} \times S^{Y}$ be an optimal strategy of $B G$ that satisfy the conditions of (1) and (2). Also $e^{T} x^{\prime}=\frac{1}{w}$ and $e^{T} y^{\prime}=\frac{1}{v}$ that is, $e^{T} x^{\prime *}=\frac{1}{w^{*}}$ and $e^{T} y^{\prime *}=\frac{1}{v^{*}}$. So the constraints of 
Theorem 8 can be written into the following form,

$$
\begin{aligned}
A y^{*} & \leq v^{*} e \quad \text { because } x^{T} A y^{*} \leq v^{*} x^{T} e=v^{*} \\
\text { Hence, } A y & \leq v e \text { or } A y^{\prime} \leq e \\
\text { i.e, } \sum_{j=1}^{n} a_{i j} y_{j}^{\prime} & \leq 1 \text { for } i=1,2, \cdots, m
\end{aligned}
$$

and

$$
\begin{aligned}
x^{* T} B & \leq w^{*} e \quad \text { because } \quad x^{* T} B y \leq w^{*} y^{T} e=w^{*} \\
\text { Hence, } B^{T} x & \leq w e \text { or } B^{T} x^{\prime} \leq e \\
\text { i.e, } \quad \sum_{i=1}^{m} b_{i j} x_{i}^{\prime} & \leq 1 \text { for } j=1,2, \cdots, n
\end{aligned}
$$

Therefore, the objective function of the Theorem $\mathbf{8}$ can be modified into the following form,

$$
\begin{gathered}
\max x^{T}(A+B) y-v-w \\
\text { or } \quad \max \left(\frac{x}{w}\right)^{T}(A+B)\left(\frac{y}{v}\right)+\frac{1}{w}+\frac{1}{v} \\
\text { Hence, } \quad \max x^{\prime T}(A+B) y^{\prime}+e^{T} x^{\prime}+e^{T} y^{\prime}
\end{gathered}
$$

Now recall the Equations (5), (8), and (11) with $x^{\prime}, y^{\prime} \geq 0$, the theorem is obvious.

\section{Bifuzzy bi-matrix game}

In the crisp scenario, there exists a beautiful relationship on bi-matrix game. It is therefore natural to ask if something similar holds in bifuzzy scenario as well. In many applied situation, the elements of the bi-matrix game are not fixed. So the elements are imprecise. Therefore, to introduce the bi-matrix game, we considered the elements as bifuzzy variables and then it is measured by Chance. Let the bifuzzy variable $\zeta_{i j}$ denote the payoff element that player I gains or the bifuzzy variable $\rho_{i j}$ be the payoff element that player II gains when players I and II play the strategies $i$ and $j$, respectively. Then, the bifuzzy bi-matrix game can be represented as follows:

$$
(\zeta, \rho)=\left(\begin{array}{cccc}
\left(\zeta_{11}, \rho_{11}\right) & \left(\zeta_{12}, \rho_{12}\right) & \ldots & \left(\zeta_{1 n}, \rho_{1 n}\right) \\
\left(\zeta_{21}, \rho_{21}\right) & \left(\zeta_{22}, \rho_{22}\right) & \ldots & \left(\zeta_{2 n}, \rho_{2 n}\right) \\
\cdot & \cdot & \ldots & \cdot \\
\cdot & \cdot & \ldots & \cdot \\
\cdot & \cdot & \ldots & \cdot \\
\left(\zeta_{m 1}, \rho_{m 1}\right) & \left(\zeta_{m 2}, \rho_{m 2}\right) & \ldots & \left(\zeta_{m n}, \rho_{m n}\right)
\end{array}\right)
$$

Definition 9. Let $\zeta_{i j}$ and $\rho_{i j}(i=1,2, \ldots, m ; j=1,2, \cdots, n)$ be independent bifuzzy variables. Then, $\left(x^{*}, y^{*}\right)$ is called an expected bifuzzy Nash equilibrium strategy to the bifuzzy bi-matrix game $\left\{S^{X}, S^{Y}, \zeta, \rho\right\}$ if,

$$
\begin{aligned}
v^{*} & =E\left[x^{* T} \zeta y^{*}\right] \geq E\left[x^{T} \zeta y^{*}\right], \forall x \in S^{X} \\
w^{*} & =E\left[x^{* T} \rho y^{*}\right] \geq E\left[x^{* T} \rho y\right], \forall y \in S^{Y}
\end{aligned}
$$

The pair $\left(v^{*}, w^{*}\right)$ is called an optimum value of the bifuzzy bi-matrix game. 
Definition 10. Let $\zeta_{i j}$ and $\rho_{i j}(i=1,2, \ldots, m ; j=1,2, \cdots, n)$ are different independent bifuzzy variables, $(\alpha, \delta) \in(0,1]$ and $w, v \in \Re$ be predetermined level of the bifuzzy payoffs. Then $\left(x^{*}, y^{*}\right)$ is called a $(\alpha, \delta)$-bifuzzy equilibrium strategy to bifuzzy bi-matrix game $\left\{S^{X}, S^{Y}, \zeta, \rho\right\}$ if,

$$
\begin{gathered}
\max \left\{v \mid \operatorname{Ch}\left\{x^{T} \zeta y^{*} \geq v\right\}(\alpha) \geq \delta\right\} \leq \max \left\{v \mid \operatorname{Ch}\left\{x^{* T} \zeta y^{*} \geq v\right\}(\alpha) \geq \delta\right\} \\
\max \left\{w \mid \operatorname{Ch}\left\{x^{* T} \rho y \geq w\right\}(\alpha) \geq \delta\right\} \leq \max \left\{w \mid \operatorname{Ch}\left\{x^{* T} \rho y^{*} \geq w\right\}(\alpha) \geq \delta\right\}
\end{gathered}
$$

Lemma 2. Let bifuzzy bi-matrix game $B G=\left\{S^{X}, S^{Y}, \zeta, \rho\right\}$ and the value of the game $v^{*}$ for player I is given by

$$
v^{*}=E\left[x^{* T} \zeta y^{*}\right]=\max _{x}\left\{x^{T} \zeta y^{*}: e^{T} x=1, x \geq 0\right\}
$$

and $w^{*}$ is the value of the game for player II is given by

$$
w^{*}=E\left[x^{* T} \rho y^{*}\right]=\max _{y}\left\{x^{* T} \rho y: e^{T} y=1, y \geq 0\right\}
$$

where the max operator is defined as,

$$
\max _{y \in S^{Y}}\left\{x^{T} \zeta y\right\}=x^{T} \zeta y_{\max } \text { such that } E\left[x^{T} \zeta y\right] \leq E\left[x^{T} \zeta y_{\max }\right]
$$

If we assume that $x_{i}^{\prime}=\frac{x_{i}}{w}$ and $y_{i}^{\prime}=\frac{y_{i}}{v}$ (for $\left.i=1,2, \cdots, m ; j=1,2, \cdots, n\right)$ and the expected value operator $(E)$ defined on the objective function and the uncertainty of the bifuzzy constraints is measured with bifuzzy measurable function Chance with confidence level $(\alpha, \delta) \in(0,1]$. Then the Lemma (1) reduces into the following quadratic programming problem $(Q P P)$ :

$$
\begin{gathered}
\max e^{T} x^{\prime}+e^{T} y^{\prime}+E\left[x^{\prime T}(\zeta+\rho) y^{\prime}\right] \\
\text { subject to, } \quad \operatorname{Ch}\left\{\sum_{j=1}^{n} \zeta_{i j} y_{j}^{\prime} \leq 1\right\}(\alpha) \geq \delta \quad \text { for } \quad i=1,2, \cdots, m \\
\operatorname{Ch}\left\{\sum_{i=1}^{m} \rho_{i j} x_{i}^{\prime} \leq 1\right\}(\alpha) \geq \delta \quad \text { for } \quad j=1,2, \cdots, n \\
y^{\prime} \geq 0, x^{\prime} \geq 0
\end{gathered}
$$

where $\left(x^{*}, y^{*}\right)$ is the equilibrium strategy and $\left(v^{*}, w^{*}\right)$ is the equilibrium outcome of the bifuzzy bi-matrix game.

Proof. Since $\left(x^{*}, y^{*}\right) \in S^{X} \times S^{Y}$ be an equilibrium strategy of the bifuzzy bi-matrix game if and only if $x^{*}$ and $y^{*}$ are simultaneously solutions of the following two problems,

$$
\left\{\begin{aligned}
\max & x^{T} \zeta y^{*} \\
\text { subject to, } & e^{T} x=1 \\
& x \geq 0 .
\end{aligned}\right.
$$

and

$$
\left\{\begin{aligned}
\max & x^{* T} \rho y \\
\text { subject to, } & e^{T} y=1 \\
& y \geq 0 .
\end{aligned}\right.
$$

Here, $\left(x^{*}, y^{*}\right) \in S^{X} \times S^{Y}$ be an arbitrary strategy of the bifuzzy bi-matrix game that satisfy the conditions (3.12) and (3.13). Also, $e^{T} x^{\prime}=\frac{1}{w}$ and $e^{T} y^{\prime}=\frac{1}{v}$, that is, $e^{T} x^{\prime *}=\frac{1}{w^{*}}$ and $e^{T} y^{\prime *}=\frac{1}{v^{*}}$. So the constraints of Lemma (1) are the elements of bifuzzy variables 
for bifuzzy bi-matrix game. So we cannot directly write the inequality for bifuzzy payoff. For the uncertainty of the payoff elements, applying the bifuzzy uncertain measurable function Chance with confidence level $(\alpha, \delta) \in(0,1]$ as follows:

$$
\begin{aligned}
& \operatorname{Ch}\left\{\zeta y^{*} \leq v^{*} e\right\}(\alpha) \geq \delta \text { because, } \operatorname{Ch}\left\{x^{T} \zeta y^{*} \leq v^{*} x^{T} e=v^{*}\right\}(\alpha) \geq \delta \\
\text { Hence, } & \operatorname{Ch}\{\zeta y \leq v e\}(\alpha) \geq \delta \text { or, } \operatorname{Ch}\left\{\zeta y^{\prime} \leq e\right\}(\alpha) \geq \delta \\
& \operatorname{Ch}\left\{\sum_{j=1}^{n} \zeta_{i j} y_{j}^{\prime} \leq 1\right\}(\alpha) \geq \delta \text { for } i=1,2, \cdots, m
\end{aligned}
$$

and

$$
\mathrm{Ch}\left\{x^{* T} \rho \leq w^{*} e\right\}(\alpha) \geq \delta \text { because, } \operatorname{Ch}\left\{x^{* T} \rho y \leq w^{*} y^{T} e=w^{*}\right\}(\alpha) \geq \delta
$$

Hence, $\operatorname{Ch}\left\{\rho^{T} x \leq w e\right\}(\alpha) \geq \delta$ or, $\operatorname{Ch}\left\{\rho^{T} x^{\prime} \leq e\right\}(\alpha) \geq \delta$

$$
\operatorname{Ch}\left\{\sum_{i=1}^{m} \rho_{i j} x_{i}^{\prime} \leq 1\right\}(\alpha) \geq \delta \quad \text { for } \quad j=1,2, \cdots, n \text {. }
$$

\section{Quadratic programming problem}

To derive the solution of the bifuzzy bi-matrix game, we are to solve the following bifuzzy quadratic programming problem,

$$
\begin{array}{cl}
\max & e^{T} x^{\prime}+e^{T} y^{\prime}+x^{\prime T}(\zeta+\rho) y^{\prime} \\
\text { subject to, } & \sum_{j=1}^{n} \zeta_{i j} y_{j}^{\prime} \leq 1 \text { for } i=1,2, \cdots, m \\
& \sum_{i=1}^{m} \rho_{i j} x_{i}^{\prime} \leq 1 \text { for } j=1,2, \cdots, n \\
& y^{\prime} \geq 0, x^{\prime} \geq 0 .
\end{array}
$$

Since the bifuzzy variables are present in the above quadratic programming problem, so traditional method is not applicable. To find the solution of the above problem, we have to introduce the bifuzzy expected operator to the objective function and the constraints, bifuzzy measurable function named as Chance with confidence level $(\alpha, \delta) \in(0,1]$. So the bifuzzy quadratic programming problem becomes

$\max E\left[e^{T} x^{\prime}+e^{T} y^{\prime}+x^{\prime T}(\zeta+\rho) y^{\prime}\right]$

subject to, $\operatorname{Ch}\left\{\sum_{j=1}^{n} \zeta_{i j} y_{j}^{\prime} \leq 1\right\}(\alpha) \geq \delta \quad$ for $\quad i=1,2, \cdots, m$

$$
\begin{aligned}
& \operatorname{Ch}\left\{\sum_{i=1}^{m} \rho_{i j} x_{i}^{\prime} \leq 1\right\}(\alpha) \geq \delta \text { for } \quad j=1,2, \cdots, n \\
& y^{\prime} \geq 0, x^{\prime} \geq 0 .
\end{aligned}
$$


With the help of Theorem 7, we can easily find that $h^{+}(x)=x^{\prime}, h^{+}(y)=y^{\prime}$, and $h^{-}(x)=0, h^{-}(y)=0$ because $y^{\prime} \geq 0, x^{\prime} \geq 0$, and using the bifuzzy set theory, the above problem can be written into crisp quadratic programming problem which depends upon the confidence level $(\alpha, \delta) \in(0,1]$, as follows:

$$
\begin{aligned}
\max \frac{1}{16} \sum_{i=1}^{m} \sum_{j=1}^{n}\left\{a_{i j 11}+a_{i j 12}+a_{i j 21}+a_{i j 22}+b_{i j 11}+b_{i j 12}+b_{i j 21}+b_{i j 22}\right. \\
+c_{i j 11}+c_{i j 12}+c_{i j 21}+c_{i j 22}+d_{i j 11}+d_{i j 12}+d_{i j 21}+d_{i j 22} \\
+x_{i j 11}+x_{i j 12}+x_{i j 21}+x_{i j 22}+y_{i j 11}+y_{i j 12}+y_{i j 21}+y_{i j 22} \\
\left.+z_{i j 11}+z_{i j 12}+z_{i j 21}+z_{i j 22}+t_{i j 11}+t_{i j 12}+t_{i j 21}+t_{i j 22}\right\} x_{i}^{\prime} y_{j}^{\prime} \\
+\sum_{i=1}^{m} x_{i}^{\prime}+\sum_{j=1}^{n} y_{j}^{\prime}
\end{aligned}
$$

subject to,

If $\delta<0.5, \alpha<0.5$,

$$
\begin{array}{r}
\sum_{j=1}^{n}\left\{(1-2 \delta)\left\{(1-2 \alpha) a_{i j 11}+2 \alpha b_{i j 11}\right\}+2 \delta\left\{(1-2 \alpha) a_{i j 12}+2 \alpha b_{i j 12}\right\}\right\} y_{j}^{\prime} \leq 1 \\
\text { for } i=1,2, \cdots, m \\
\sum_{i=1}^{m}\left\{(1-2 \delta)\left\{(1-2 \alpha) x_{i j 11}+2 \alpha y_{i j 11}\right\}+2 \delta\left\{(1-2 \alpha) x_{i j 12}+2 \alpha y_{i j 12}\right\}\right\} x_{i}^{\prime} \leq 1 \\
\text { for } j=1,2, \cdots, n \\
y^{\prime} \geq 0, x^{\prime} \geq 0
\end{array}
$$

If $\delta<0.5, \alpha \geq 0.5$,

$$
\begin{array}{r}
\sum_{j=1}^{n}\left\{(1-2 \delta)\left\{(2-2 \alpha) c_{i j 11}+(2 \alpha-1) d_{i j 11}\right\}+2 \delta\left\{(2-2 \alpha) c_{i j 12}+(2 \alpha-1) d_{i j 12}\right\}\right\} y_{j}^{\prime} \leq 1 \\
\text { for } i=1,2, \cdots, m \\
\sum_{i=1}^{m}\left\{(1-2 \delta)\left\{(2-2 \alpha) z_{i j 11}+(2 \alpha-1) t_{i j 11}\right\}+2 \delta\left\{(2-2 \alpha) z_{i j 12}+(2 \alpha-1) t_{i j 12}\right\}\right\} x_{i}^{\prime} \leq 1 \\
\text { for } j=1,2, \cdots, n \\
y^{\prime} \geq 0, x^{\prime} \geq 0
\end{array}
$$

If $\delta \geq 0.5, \alpha<0.5$,

$$
\begin{array}{r}
\sum_{j=1}^{n}\left\{(2-2 \delta)\left\{(1-2 \alpha) a_{i j 21}+2 \alpha b_{i j 21}\right\}+(2 \delta-1)\left\{(1-2 \alpha) a_{i j 22}+2 \alpha b_{i j 22}\right\}\right\} y_{j}^{\prime} \leq 1 \\
\text { for } i=1,2, \cdots, m \\
\sum_{i=1}^{m}\left\{(2-2 \delta)\left\{(1-2 \alpha) x_{i j 21}+2 \alpha y_{i j 21}\right\}+(2 \delta-1)\left\{(1-2 \alpha) x_{i j 22}+2 \alpha y_{i j 22}\right\}\right\} x_{i}^{\prime} \leq 1 \\
\text { for } j=1,2, \cdots, n \\
y^{\prime} \geq 0, x^{\prime} \geq 0
\end{array}
$$


If $\delta \geq 0.5, \alpha \geq 0.5$,

$$
\begin{array}{r}
\sum_{j=1}^{n}\left\{(2-2 \delta)\left\{(2-2 \alpha) c_{i j 21}+(2 \alpha-1) d_{i j 21}\right\}+(2 \delta-1)\left\{(2-2 \alpha) c_{i j 22}+\right.\right. \\
\left.\left.(2 \alpha-1) d_{i j 22}\right\}\right\} y_{j}^{\prime} \leq 1 \quad \text { for } i=1,2, \cdots, m \\
\sum_{i=1}^{m}\left\{(2-2 \delta)\left\{(2-2 \alpha) z_{i j 21}+(2 \alpha-1) t_{i j 21}\right\}+(2 \delta-1)\left\{(2-2 \alpha) z_{i j 22}+\right.\right. \\
\left.\left.(2 \alpha-1) t_{i j 22}\right\}\right\} x_{i}^{\prime} \leq 1 \quad \text { for } j=1,2, \cdots, n \\
y^{\prime} \geq 0, \quad x^{\prime} \geq 0
\end{array}
$$

where the bifuzzy variable for player I's payoff is $\zeta=\left(\zeta_{i j a}, \zeta_{i j b}, \zeta_{i j c}, \zeta_{i j c}\right)$ and for player II's payoff is $\rho=\left(\rho_{i j x}, \rho_{i j y}, \rho_{i j z}, \rho_{i j t}\right)$ with fuzzy variables defined as, $\zeta_{i j a}=$ $\left(a_{i j 11}, b_{i j 11}, c_{i j 11}, d_{i j 11}\right), \zeta_{i j b}=\left(a_{i j 12}, b_{i j 12}, c_{i j 12}, d_{i j 12}\right), \zeta_{i j c}=\left(a_{i j 21}, b_{i j 21}, c_{i j 21}, d_{i j 21}\right), \zeta_{i j d}=$ $\left(a_{i j 22}, b_{i j 22}, c_{i j 22}, d_{i j 22}\right)$ and $\rho_{i j x}=\left(x_{i j 11}, y_{i j 11}, z_{i j 11}, t_{i j 11}\right), \rho_{i j y}=\left(x_{i j 12}, y_{i j 12}, z_{i j 12}, t_{i j 12}\right), \rho_{i j z}=$ $\left(x_{i j 21}, y_{i j 21}, z_{i j 21}, t_{i j 21}\right), \rho_{i j t}=\left(x_{i j 22}, y_{i j 22}, z_{i j 22}, t_{i j 22}\right)$

Depending upon confidence level $(\delta, \alpha) \in(0,1]$, the proposed bifuzzy bi-matrix game becomes a standard quadratic programming problem with crisp constraints and obtained the values of bifuzzy bi-matrix game $w$ and $v$ to the corresponding strategies of the players, using Wolfe's modified simplex method.

\section{A numerical example}

A bifuzzy bi-matrix game problem has been formulated for two mobile network companies at West Bengal in India, depending upon their profits earned. It is considered that both the companies have four plans for rate of call charges. The plans are 1 paisa/s, 30 paisa/min, 50 paisa/2 min, and 3 rupees/10 min for their networks. Due to the availability of network and depending upon the plan, assuming that the people talk more in the first plan rather than the second plan. So, the profit occurs more in the first plan as compared to other plan. Suppose for the first company, the profit of the plan-a (1 paisa/s) is about 180 to 190 million per hour, but at night time, the profit arises up to 195 million and during morning it reduces to 175 million. For the plan-b (30 paisa/min), profit is about 156 to 158 million per hour, but at night time, the profit arises up to 160 million and during morning, it reduces to 150 million. For the plan-c (50 paisa/2 min), profit is about 90 to 95 million per hour, but at night time, the profit arises up to 100 million and during morning, it reduces to 80 million. For the plan-d ( 3 rupees/10 min), profit is about 120 to 130 million per hour, but at night time, the profit arises up to 140 million and during morning, it reduces to 100 million. Similarly, profit of the second company for plan-a ( 1 paisa/s) is about 165 to 170 million per hour, but at night time, the profit arises up to 175 million and during morning, it reduces to 160 million. For the plan-b (30 paisa/min), profit is about 145 to 148 million per hour, but at night time, the profit arises up to 150 million and during morning, it reduces to 140 million. For the plan-c (50 paisa/2 $\mathrm{min}$ ), profit is about 75 to 78 million per hour, but at night time, the profit arises up to 82 million and during morning, it reduces to 70 million. For the plan-d (3 rupees/10 min), profit is about 100 to 110 million per hour but at night time, the profit arises up to 130 million and during morning, it reduces to 90 million. For this marketing strategy, the first company losses 2 million during at morning time and gains 2 million during at night time and other times gain varies between -1 and 1 million per hour. Similarly, the second com- 
pany losses 3 million during morning and gains 2 million during at night time and other times gain varies between -2 and 1 million per hour.

To optimize their profits, a bifuzzy bi-matrix game is formulated whose payoff matrix elements are bifuzzy variables representing the profit of the company per hour. For the first company, bifuzzy variable $\zeta_{i j}=\left(\xi_{i j}-2, \xi_{i j}-1, \xi_{i j}+1, \xi_{i j}+2\right)$, and for second company, bifuzzy variable $\rho_{i j}=\left(\varrho_{i j}-3, \varrho_{i j}-2, \varrho_{i j}+1, \varrho_{i j}+2\right)$ with their fuzzy variables $\xi_{i j}$ and $\varrho_{i j}$, respectively. For the first company, fuzzy variables for plan-a is $\xi_{11}=(175,180,190,195)$, plan-b $\xi_{12}=(150,156,158,160)$, plan-c $\xi_{21}=(80,90,95,100)$, and plan-d $\xi_{22}=$ $(100,120,130,140)$. Similarly for the second company, fuzzy variables for the plan-a is $\varrho_{11}=(160,165,170,175)$, plan-b $\varrho_{12}=(140,145,148,150)$, plan-c $\varrho_{21}=(70,75,78,82)$, and plan-d $\varrho_{22}=(90,100,110,130)$. Here, one of the models is shown for particular case, where values of the confidence levels $\delta=\frac{3}{4}$ and $\alpha=\frac{1}{4}$ (choice of decision maker) are considered. Applying the proposed methodology discussed in earlier section, the equivalent crisp quadratic programming problem for the bifuzzy bi-matrix game is constructed as follows:

$$
\begin{aligned}
\max 309.625000 x_{1}^{\prime} y_{1}^{\prime}+264.375000 x_{1}^{\prime} y_{2}^{\prime}+147.625000 x_{2}^{\prime} y_{1}^{\prime} & +202.125000 x_{2}^{\prime} y_{2}^{\prime} \\
& +x_{1}^{\prime}+x_{2}^{\prime}+y_{1}^{\prime}+y_{2}^{\prime} \\
179.000000 y_{1}^{\prime}+154.500000 y_{2}^{\prime} & \leq 1 \\
86.500000 y_{1}^{\prime}+111.500000 y_{2}^{\prime} & \leq 1 \\
164.000000 x_{1}^{\prime}+74.000000 x_{2}^{\prime} & \leq 1 \\
144.000000 x_{1}^{\prime}+96.500000 x_{2}^{\prime} & \leq 1 \\
y_{1}^{\prime}, y_{2}^{\prime}, x_{1}^{\prime}, x_{2}^{\prime} & \geq 0
\end{aligned}
$$

The solution of the above non-linear programming problem is

$$
y^{\prime}=(0.0,0.006472492) \text { and } x^{\prime}=(0.0,0.002072539)
$$

which leads to the following solution of the original problem

$$
\begin{aligned}
& y^{*}=(0,1) \text { and } w=482.499969 \\
& x^{*}=(0,1) \text { and } v=154.500000 .
\end{aligned}
$$

Now for different values of confidence levels (Cofd. lev.), the values of the game (Val. game) and the strategies (Str.) of the players I and II are presented in Table 1.

From the above tabulated values, it is observed that the values of the game increase with increasing the values of confidence levels. For particular case, when $\delta=1, \alpha=1$, the values of the game are maximum, and consequently, it can be concluded that both the companies have maximum profit. Again for $\delta=0.1, \alpha=0.1$, the values of the game are minimum and so the companies have minimum profit. Also, the decision maker (here company) has the right to choose the confidence levels. So for the selection of proper network and time condition, the companies have chosen the confidence levels and obtained the values of the game, i.e., the profits of the companies are maximum and appropriate. 
Table 1 Values of conf. levels, values of game, and strategies of the players

\begin{tabular}{|c|c|c|c|c|c|c|c|}
\hline $\begin{array}{c}\text { Cofd. lev. } \\
\delta\end{array}$ & $\begin{array}{c}\text { Cofd. lev. } \\
\alpha\end{array}$ & $\begin{array}{c}\text { Val. game II } \\
\qquad w\end{array}$ & $\begin{array}{c}\text { Val. game I } \\
v\end{array}$ & $\begin{array}{c}\text { Str. II } \\
y 1\end{array}$ & $\begin{array}{c}\text { Str. II } \\
\text { y2 }\end{array}$ & $\begin{array}{c}\text { Str. I } \\
x 1\end{array}$ & $\begin{array}{c}\text { Str. } 1 \\
x 2\end{array}$ \\
\hline 0.100000 & 0.100000 & 102.199997 & 149.399994 & 0.000000 & 1.000000 & 0.000000 & 1.000000 \\
\hline 0.100000 & 0.200000 & 106.200005 & 150.600006 & 0.000000 & 1.000000 & 0.000000 & 1.000000 \\
\hline 0.100000 & 0.300000 & 110.199997 & 151.800018 & 0.000000 & 1.000000 & 0.000000 & 1.000000 \\
\hline 0.100000 & 0.400000 & 114.199997 & 153.000000 & 0.000000 & 1.000000 & 0.000000 & 1.000000 \\
\hline 0.100000 & 0.500000 & 128.199997 & 156.199982 & 0.000000 & 1.000000 & 0.000000 & 1.000000 \\
\hline 0.100000 & 0.600000 & 130.199997 & 156.599991 & 0.000000 & 1.000000 & 0.000000 & 1.000000 \\
\hline 0.100000 & 0.700000 & 132.199997 & 157.000000 & 0.000000 & 1.000000 & 0.000000 & 1.000000 \\
\hline 0.100000 & 0.800000 & 146.478531 & 157.400009 & 0.000000 & 1.000000 & 0.529248 & 0.470752 \\
\hline 0.100000 & 0.900000 & 147.676834 & 157.800018 & 0.000000 & 1.000000 & 0.531335 & 0.468665 \\
\hline 0.100000 & 1.000000 & 148.866653 & 158.199982 & 0.000000 & 1.000000 & 0.533333 & 0.466667 \\
\hline 0.200000 & 0.100000 & 102.400002 & 149.600006 & 0.000000 & 1.000000 & 0.000000 & 1.000000 \\
\hline 0.200000 & 0.200000 & 106.400002 & 150.800003 & 0.000000 & 1.000000 & 0.000000 & 1.000000 \\
\hline 0.200000 & 0.300000 & 110.400002 & 152.000015 & 0.000000 & 1.000000 & 0.000000 & 1.000000 \\
\hline 0.200000 & 0.400000 & 114.400002 & 153.199997 & 0.000000 & 1.000000 & 0.000000 & 1.000000 \\
\hline 0.200000 & 0.500000 & 128.399994 & 156.399994 & 0.000000 & 1.000000 & 0.000000 & 1.000000 \\
\hline 0.200000 & 0.600000 & 130.399994 & 156.800003 & 0.000000 & 1.000000 & 0.000000 & 1.000000 \\
\hline 0.200000 & 0.700000 & 132.399994 & 157.200012 & 0.000000 & 1.000000 & 0.000000 & 1.000000 \\
\hline 0.200000 & 0.800000 & 146.678558 & 157.600021 & 0.000000 & 1.000000 & 0.529248 & 0.470752 \\
\hline 0.200000 & 0.900000 & 147.876846 & 158.000000 & 0.000000 & 1.000000 & 0.531335 & 0.468665 \\
\hline 0.200000 & 1.000000 & 149.066666 & 158.399979 & 0.000000 & 1.000000 & 0.533333 & 0.466667 \\
\hline 0.300000 & 0.100000 & 102.599998 & 149.800003 & 0.000000 & 1.000000 & 0.000000 & 1.000000 \\
\hline 0.300000 & 0.200000 & 106.599998 & 150.999985 & 0.000000 & 1.000000 & 0.000000 & 1.000000 \\
\hline 0.300000 & 0.300000 & 110.600006 & 152.200012 & 0.000000 & 1.000000 & 0.000000 & 1.000000 \\
\hline 0.300000 & 0.400000 & 110.600006 & 152.200012 & 0.000000 & 1.000000 & 0.000000 & 1.000000 \\
\hline 0.300000 & 0.500000 & 128.600006 & 156.599991 & 0.000000 & 1.000000 & 0.000000 & 1.000000 \\
\hline 0.300000 & 0.600000 & 130.600021 & 157.000000 & 0.000000 & 1.000000 & 0.000000 & 1.000000 \\
\hline 0.300000 & 0.700000 & 132.600006 & 157.400009 & 0.000000 & 1.000000 & 0.000000 & 1.000000 \\
\hline 0.300000 & 0.800000 & 146.878555 & 157.800018 & 0.000000 & 1.000000 & 0.529248 & 0.470752 \\
\hline 0.300000 & 0.900000 & 148.076859 & 158.199982 & 0.000000 & 1.000000 & 0.531335 & 0.468665 \\
\hline 0.300000 & 1.000000 & 149.266678 & 158.600006 & 0.000000 & 1.000000 & 0.533333 & 0.466667 \\
\hline 0.400000 & 0.100000 & 102.800003 & 150.000000 & 0.000000 & & & \\
\hline 0.400000 & 0.200000 & 106.799995 & 151.199997 & 0.000000 & 1.000000 & 0.000000 & 1.000000 \\
\hline 0.400000 & 0.300000 & 110.799995 & 152.399994 & 0.000000 & 1.000000 & 0.000000 & 1.000000 \\
\hline 0.400000 & 0.400000 & 114.800011 & 153.600021 & 0.000000 & 1.000000 & 0.000000 & 1.000000 \\
\hline 0.400000 & 0.500000 & 128.800003 & 156.800003 & 0.000000 & 1.000000 & 0.000000 & 1.000000 \\
\hline 0.400000 & 0.600000 & 130.800003 & 157.200012 & 0.000000 & 1.000000 & 0.000000 & 1.000000 \\
\hline 0.400000 & 0.700000 & 132.800003 & 157.600021 & 0.000000 & 1.000000 & 0.000000 & 1.000000 \\
\hline 0.400000 & 0.800000 & 147.078552 & 158.000000 & 0.000000 & 1.000000 & 0.529248 & 0.470752 \\
\hline 0.400000 & 0.900000 & 148.276825 & 158.399979 & 0.000000 & 1.000000 & 0.531335 & 0.468665 \\
\hline 0.400000 & 1.000000 & 149.466675 & 158.800003 & 0.000000 & 1.000000 & 0.533333 & 0.466667 \\
\hline 0.500000 & 0.100000 & 104.999992 & 152.200012 & 0.000000 & 1.000000 & 0.000000 & 1.000000 \\
\hline 0.500000 & 0.200000 & 108.999992 & 153.399994 & 0.000000 & 1.000000 & 0.000000 & 1.000000 \\
\hline 0.500000 & 0.300000 & 112.999985 & 154.600006 & 0.000000 & 1.000000 & 0.000000 & 1.000000 \\
\hline 0.500000 & 0.400000 & 116.999992 & 155.800003 & 0.000000 & 1.000000 & 0.000000 & 1.000000 \\
\hline 0.500000 & 0.500000 & 131.000000 & 159.000000 & 0.000000 & 1.000000 & 0.000000 & 1.000000 \\
\hline 0.500000 & 0.600000 & 133.000000 & 159.399994 & 0.000000 & 1.000000 & 0.000000 & 1.000000 \\
\hline 0.500000 & 0.700000 & 135.000015 & 159.800018 & 0.000000 & 1.000000 & 0.000000 & 1.000000 \\
\hline 0.500000 & 0.800000 & 149.278549 & 160.200012 & 0.000000 & 1.000000 & 0.529248 & 0.470752 \\
\hline 0.500000 & 0.900000 & 150.476837 & 160.600006 & 0.000000 & 1.000000 & 0.531335 & 0.468665 \\
\hline 0.500000 & 1.000000 & 151.666656 & 161.000015 & 0.000000 & 1.000000 & 0.533333 & 0.466667 \\
\hline
\end{tabular}


Table 1 Values of conf. levels, values of game, and strategies of the players (Continued)

\begin{tabular}{|c|c|c|c|c|c|c|c|}
\hline 0.600000 & 0.100000 & 105.199989 & 152.399994 & 0.000000 & 1.000000 & 0.000000 & 1.000000 \\
\hline 0.600000 & 0.200000 & 109.200005 & 153.600021 & 0.000000 & 1.000000 & 0.000000 & 1.000000 \\
\hline 0.600000 & 0.300000 & 113.199997 & 154.800003 & 0.000000 & 1.000000 & 0.000000 & 1.000000 \\
\hline 0.600000 & 0.400000 & 117.200012 & 156.000015 & 0.000000 & 1.000000 & 0.000000 & 1.000000 \\
\hline 0.600000 & 0.500000 & 131.200012 & 159.199997 & 0.000000 & 1.000000 & 0.000000 & 1.000000 \\
\hline 0.600000 & 0.600000 & 133.199982 & 159.600006 & 0.000000 & 1.000000 & 0.000000 & 1.000000 \\
\hline 0.600000 & 0.700000 & 135.199997 & 160.000000 & 0.000000 & 1.000000 & 0.000000 & 1.000000 \\
\hline 0.600000 & 0.800000 & 149.478561 & 160.399994 & 0.000000 & 1.000000 & 0.529248 & 0.470752 \\
\hline 0.600000 & 0.900000 & 150.676849 & 160.800003 & 0.000000 & 1.000000 & 0.531335 & 0.468665 \\
\hline 0.600000 & 1.000000 & 151.866684 & 161.199997 & 0.000000 & 1.000000 & 0.533333 & 0.466667 \\
\hline 0.700000 & 0.100000 & 151.866684 & 161.199997 & 0.000000 & 1.000000 & 0.533333 & 0.466667 \\
\hline 0.700000 & 0.200000 & 109.399994 & 153.800018 & 0.000000 & 1.000000 & 0.000000 & 1.000000 \\
\hline 0.700000 & 0.300000 & 113.399994 & 155.000000 & 0.000000 & 1.000000 & 0.000000 & 1.000000 \\
\hline 0.700000 & 0.400000 & 117.400002 & 156.199982 & 0.000000 & 1.000000 & 0.000000 & 1.000000 \\
\hline 0.700000 & 0.500000 & 131.400009 & 159.399994 & 0.000000 & 1.000000 & 0.000000 & 1.000000 \\
\hline 0.700000 & 0.600000 & 133.399994 & 159.800018 & 0.000000 & 1.000000 & 0.000000 & 1.000000 \\
\hline 0.700000 & 0.700000 & 135.399994 & 160.200012 & 0.000000 & 1.000000 & 0.000000 & 1.000000 \\
\hline 0.700000 & 0.800000 & 149.678543 & 160.600006 & 0.000000 & 1.000000 & 0.529248 & 0.470752 \\
\hline 0.700000 & 0.900000 & 150.876831 & 161.000015 & 0.000000 & 1.000000 & 0.531335 & 0.468665 \\
\hline 0.700000 & 1.000000 & 152.066666 & 161.399994 & 0.000000 & 1.000000 & 0.533333 & 0.466667 \\
\hline 0.800000 & 0.100000 & 105.599998 & 152.800018 & 0.000000 & 1.000000 & 0.000000 & 1.000000 \\
\hline 0.800000 & 0.200000 & 105.599998 & 152.800018 & 0.000000 & 1.000000 & 0.000000 & 1.000000 \\
\hline 0.800000 & 0.300000 & 113.599998 & 155.199997 & 0.000000 & 1.000000 & 0.000000 & 1.000000 \\
\hline 0.800000 & 0.400000 & 117.599998 & 156.399994 & 0.000000 & 1.000000 & 0.000000 & 1.000000 \\
\hline 0.800000 & 0.500000 & 131.600006 & 159.600006 & 0.000000 & 1.000000 & 0.000000 & 1.000000 \\
\hline 0.800000 & 0.600000 & 133.599991 & 160.000000 & 0.000000 & 1.000000 & 0.000000 & 1.000000 \\
\hline 0.800000 & 0.700000 & 135.600006 & 160.399994 & 0.000000 & 1.000000 & 0.000000 & 1.000000 \\
\hline 0.800000 & 0.800000 & 149.878540 & 160.800003 & 0.000000 & 1.000000 & 0.529248 & 0.470752 \\
\hline 0.800000 & 0.900000 & 151.076843 & 161.199997 & 0.000000 & 1.000000 & 0.531335 & 0.468 \\
\hline 0.800000 & 1.000000 & 152.266678 & 161.600006 & 0.000000 & 1.000000 & 0.533333 & 0.466667 \\
\hline 0.900000 & 0.100000 & 105.799995 & 153.000000 & 0.000000 & 1.000000 & 0.000000 & 1.000000 \\
\hline 0.900000 & 0.200000 & 109.800003 & 154.200012 & 0.000000 & 1.000000 & 0.000000 & 1.000000 \\
\hline 0.900000 & 0.300000 & 113.800003 & 155.399994 & 0.000000 & 1.000000 & 0.000000 & 1.000000 \\
\hline 0.900000 & 0.400000 & 117.800003 & 156.599991 & 0.000000 & 1.000000 & 0.000000 & 1.000000 \\
\hline 0.900000 & 0.500000 & 131.800003 & 159.800018 & 0.000000 & 1.000000 & 0.000000 & 1.000000 \\
\hline 0.900000 & 0.600000 & 133.800018 & 160.200012 & 0.000000 & 1.000000 & 0.000000 & 1.000000 \\
\hline 0.900000 & 0.700000 & 135.800003 & 160.600006 & 0.000000 & 1.000000 & 0.000000 & 1.000000 \\
\hline 0.900000 & 0.800000 & 150.078537 & 161.000015 & 0.000000 & 1.000000 & 0.529248 & 0.470752 \\
\hline 0.900000 & 0.900000 & 151.276840 & 161.399994 & 0.000000 & 1.000000 & 0.531335 & 0.468665 \\
\hline 0.900000 & 1.000000 & 152.466690 & 161.800003 & 0.000000 & 1.000000 & 0.533333 & 0.466667 \\
\hline 1.000000 & 0.100000 & 106.000000 & 153.199997 & 0.000000 & 1.000000 & 0.000000 & 1.000000 \\
\hline 1.000000 & 0.200000 & 110.000000 & 154.399994 & 0.000000 & 1.000000 & 0.000000 & 1.000000 \\
\hline 1.000000 & 0.300000 & 114.000000 & 155.600006 & 0.000000 & 1.000000 & 0.000000 & 1.000000 \\
\hline 1.000000 & 0.400000 & 118.000000 & 156.800003 & 0.000000 & 1.000000 & 0.000000 & 1.000000 \\
\hline 1.000000 & 0.500000 & 132.000000 & 160.000000 & 0.000000 & 1.000000 & 0.000000 & 1.000000 \\
\hline 1.000000 & 0.600000 & 134.000000 & 160.399994 & 0.000000 & 1.000000 & 0.000000 & 1.000000 \\
\hline 1.000000 & 0.700000 & 136.000000 & 160.800003 & 0.000000 & 1.000000 & 0.000000 & 1.000000 \\
\hline 1.000000 & 0.800000 & 150.278564 & 161.199997 & 0.000000 & 1.000000 & 0.529248 & 0.470752 \\
\hline 1.000000 & 0.900000 & 151.476852 & 161.600006 & 0.000000 & 1.000000 & 0.531335 & 0.468665 \\
\hline 1.000000 & 1.000000 & 152.666641 & 161.999985 & 0.000000 & 1.000000 & 0.533333 & 0.466667 \\
\hline
\end{tabular}




\section{Conclusion}

The properties of bifuzzy variable and bi-matrix game theory have been investigated in the paper. Using bifuzzy theory, the procedure to solve the bifuzzy bi-matrix game problem has been discussed. A real-life practical problem for bifuzzy bi-matrix game has been included and solved using proposed method. Various solutions have provided for different confidence levels. The players have freedom to choose the appropriate confidence levels, and for inexact confidence levels, the solution of the bifuzzy bi-matrix game problem may not be optimum. For appropriate choice of confidence levels, the players have optimum values with strategies of the bifuzzy bi-matrix game. The present method can be used as a powerful decision-making tool for decision maker to take right decision for competitive systems.

\section{Acknowledgements}

The authors are grateful to acknowledge the anonymous referees for their helpful suggestions and comments which have led to an improvement in the quality of the paper.

\section{Author details}

${ }^{1}$ Department of Applied Mathematics with Oceanology and Computer Programming, Vidyasagar University, Midnapore, West Bengal 721102, India. ${ }^{2}$ ISRO Satellite Centre, Old Airport Road, Vimanapura Post, Bangalore 560017, India.

Received: 6 May 2013 Accepted: 19 October 2013

Published: 11 November 2013

\section{References}

1. Owen, G: Game Theory, Academic, San Diego (1995)

2. Zadeh, LA: Fuzzy sets. Inf. Control. 8, 338-353 (1965)

3. Bector, CR, Chandra, S: Fuzzy Mathematical Programming and Fuzzy Matrix Games. Springer, Berlin, Heidelberg (2005) Nishizaki, I, Sakawa, M: Fuzzy and Multiobjective Games for Conflict Resolution. Physica, Heidelberg (2001)

5. Campos, L: Fuzzy linear programming models to solve fuzzy matrix game. Fuzzy Sets Syst. 32, 275-289 (1989)

6. Roy, SK, Mula, P, Mondal, SN: A new solution concept in credibilistic game. CiiT Int. J. Fuzzy Syst. 3, 115-120 (2011)

7. Roy, SK: Game Theory Under MCDM and Fuzzy Set Theory. VDM (Verlag Dr. Muller), Germany (2010)

8. Mula, P, Roy, SK: Credibilistic bi-matrix game. J. Uncertain Syst. 6(1), 71-80 (2012)

9. Liu, B: Theory and Practice of Uncertain Programming. Physica, Heidelberg (2002)

10. Mula, P, Roy, SK: Bifuzzy matrix game. CiiT. Int. J. Fuzzy Syst. 5(1), 10-18 (2013)

11. Zhou, J, Liu, B: Analysis and algorithms of bifuzzy systems. Int. J. Uncertainty, Fuzziness Knowledge-Based Syst. 12(3), 357-376 (2004)

12. Li, X, Liu, B: A sufficient and necessary condition for credibility measures. Int. Jour. Uncert. Fuzz. Know. Based Sys. 14(5), 527-535 (2006)

13. Liu, B, Lui, YK: Expected value of fuzzy variable and fuzzy expected value models. IEEE Trans. Fuzzy Syst. 10(4), 445-450 (2002)

14. Basar, T, Olsder, GJ: Dynamic Noncooperative Game Theory. Second Edition. Academic, San Diego (1995)

\section{Submit your manuscript to a SpringerOpen ${ }^{\circ}$ journal and benefit from:}

- Convenient online submission

- Rigorous peer review

- Immediate publication on acceptance

- Open access: articles freely available online

- High visibility within the field

- Retaining the copyright to your article

Submit your next manuscript at springeropen.com 